\title{
3D/BIELA AND THE ANDROMEDIDS: FRAGMENTING VERSUS SUBLIMATING COMETS
}

\author{
P. Jenniskens ${ }^{1}$ and J. Vaubaillon ${ }^{2}$ \\ Received 2007 January 3; accepted 2007 April 22
}

\begin{abstract}
Comet 3D/Biela broke up in 1842/1843 and continued to disintegrate in the returns of 1846 and 1852 . When meteor storms were observed in November of 1872 and 1885, it was surmised that those showers were the debris from that breakup. This could have come from one of two sources: (1) the initial separation of fragments near aphelion or (2) the continued disintegration of the fragments afterward. Alternatively, the meteoroids could simply have come from water vapor drag when the fragments approached perihelion (option 3). We investigated the source of the Andromedid storms by calculating the dynamical evolution of dust ejected in a normal manner by water vapor drag in the returns from 1703 to 1866 , assuming that the comet would have remained similarly active over each return. In addition, we simulated the isotropic ejection of dust during the initial fragmentation event at aphelion in December of 1842. We conclude that option 2 is the most likely source of meteoroids encountered during the 1872 and 1885 storms, but this accounts for only a relatively small amount of mass lost in a typical comet breakup.
\end{abstract}

Key words: comets: individual (3D/Biela) - meteors, meteoroids - minor planets, asteroids

\section{INTRODUCTION}

Ever since Whipple (1951) showed that water vapor can accelerate dust particles away from a solid comet nucleus, it has been assumed that meteoroid streams are created in this manner (Williams 2004). The problem with this theory is that most of our meteor showers have no known active parent comet.

In recent years we have demonstrated that the Quadrantid and Phoenicid showers were generated in a comet fragmentation event (Jenniskens 2004; Jenniskens \& Lyytinen 2005) and concluded from the prevalence of meteor showers with no known parent bodies that comet fragmentation is likely the dominant mechanism for creating comet dust in Earth's path (Jenniskens 2006). In most cases studied to date, about as much mass was shed in the form of meteoroids as was left in the remaining body (Table 1). The Andromedids are an exception, with much less mass in the stream $\left(\sim 3.3 \times 10^{10} \mathrm{~kg}\right.$, according to Jenniskens 1995$)$ than the mass of either of the two fragments of comet Biela $\left(\sim 1400 \times 10^{10} \mathrm{~kg}\right)$. This prompted us to investigate the time and method of origin of the Andromedid dust and the prior estimation of the mass of meteoroids in the stream.

Another reason for scrutiny is the similar appearance of the breakup of comet 3D/Biela to that of the 1995 breakup of active comet 73P/Schwassmann-Wachmann 3. SchwassmannWachmann 3 broke into more than 68 fragments, with two dominant pieces called " $\mathrm{C}$ " (the primary) and " $\mathrm{B}$ " (about onethird the size of $C$ ). They were well observed in the return of 2006 and are now a topic of considerable interest. Meteoroids are expected to move into Earth's path in 2022 (Wiegert et al. 2005).

We investigate here whether the Andromedids could have originated from (1) the initial separation of fragments near aphelion or (2) the continued disintegration of the fragments afterward. Alternatively, the meteoroids could simply have come from water vapor drag when the fragments approached perihelion (option 3).

\footnotetext{
${ }^{1}$ SETI Institute, 515 North Whisman Road, Mountain View, CA 94043, USA; pjenniskens@mail.arc.nasa.gov.

${ }^{2}$ California Institute of Technology, 1200 East California Boulevard, Pasadena, CA 91125, USA.
}

\section{THE COMET AND ITS SHOWER}

\subsection{The Parent Comet}

Comet 3D/Biela (Table 2) was discovered with an absolute brightness of $H_{10}=+7.5$ mag by J. L. Montaigne of Límoges, France, on 1772 March 8.79 (Kronk 1999). It was lost for four orbits before the 1805/1806 apparition, when it passed at a distance of only $0.0366 \mathrm{AU}$, permitting the calculation of an elliptical orbit. The comet was lost for another two returns before being recovered by W. von Biela (Josephstadt, Austria) during the 1826 return. Now based on an ephemeris of the orbit, it was again seen in 1832, 1846, and 1852. The orbital period (during the 1772 return) was ultimately determined to be $P=6.87 \mathrm{yr}$ (Marsden 1972, pp. 14 and 40).

Marsden \& Sekanina (1971) investigated the nongravitational forces on Biela's orbit and concluded that Biela broke into at least two fragments in 1842 or early 1843 with a relative speed (in a sunward direction) of about $0.7 \mathrm{~m} \mathrm{~s}^{-1}$ if the breakup happened in 1842 January, or $1.4 \mathrm{~m} \mathrm{~s}^{-1}$ if the breakup occurred in 1843 July. The fragments were seen as individual comets in the returns of 1846 and 1852. Interestingly, both components were about identical in brightness $\left(H_{10}=+8.0\right.$ in $1845-1846$ and $H_{10}=+8.5$ and $<+8.5$ in 1852; Holetschek 1909). Earlier, Hubbard (1854) had the point of breakup as 1844 September, but he had incorrectly assigned the primary component A of 1846 to the northwestern component (B) instead of the southeastern component (A) in 1852 (Fig. 1). The initial breakup in 1842/1843 did not make the comet significantly brighter in the return of 1846. In earlier years the comet had been $H_{10}=+7.5$ in 1772 , $H_{10}=+9.6$ in 1805-1806 (when the comet was close to Earth), $H_{10}=+7.5$ in 1826 , and $H_{10}=+8.6$ in 1832 .

The primary fragment A continued to shed smaller pieces. Shortly after discovery on 1845 December 29 , component B was $\sim 1.5$ mag less than the primary component but continued to fluctuate in brightness, and in mid-February it briefly outshined component A before becoming fainter again. It had a sharp stellar nucleus, while the primary component was more diffuse and on occasion appeared to have multiple nuclei. At one point, as many as five fragments were seen. At least two tails developed, nearly parallel but slightly separated (Fig. 1), possibly due to the breakup of fragments some time after ejection. In late January 
TABLE 1

Documented Comet Fragmentation Events Resulting in Meteor Showers on Earth

\begin{tabular}{ccccc}
\hline \hline $\begin{array}{c}\text { Recovered } \\
\text { Fragment }\end{array}$ & $\begin{array}{c}\text { Mass } \\
\left(10^{10} \mathrm{~kg}\right)\end{array}$ & Shower & $\begin{array}{c}\text { Mass } \\
\left(10^{10} \mathrm{~kg}\right)\end{array}$ & Year \\
\hline 3D/Biela.............. & 1400 & Andromedids & $3.3^{\mathrm{a}}$ & $1842 / 1843$ \\
2003 WY25......... & 3 & Phoenicids & 10 & $\sim 1819$ \\
2003 EH1 ............ & 1600 & Quadrantids & 1000 & $\sim 1490$ \\
Marsden group ....... & 1000 & Daytime Arietids & 800 & $>1059$ \\
3200 Phaethon ....... & 6900 & Geminids & 2800 & $\sim 1030$ \\
2002 EX12 ........... & 1700 & $\alpha$ Capricornids & 520 & $\sim 10$ \\
\hline
\end{tabular}

NoTE.-From Jenniskens (2006).

${ }^{\text {a }}$ New value calculated here: $32 \times 10^{10} \mathrm{~kg}$.

and in February, when the comet approached Earth, several observers noticed an arc of light toward its companion (Challis 1846), presumably the trail of smaller fragments that was generated during the initial breakup. By mid-March, component B had faded by 3 mag relative to $A$.

The last observation of the comets was by O. W. Struve at Pulkovo Observatory on 1852 September 29. Extensive searches for the comets in 1865/1866 did not recover remaining fragments (Spratt 1984), implying that the components either faded significantly to become dormant or disintegrated completely late in the return of 1852, in the unfavorable 1859 return, or early in the return of 1866 .

At least one other comet is in an orbit similar to that of 3D/Biela (Hergenrother et al. 2001): comet P/2001 J1 (NEAT), which will return favorably in 2008 . The orbit is sufficiently different, however, to not be a remnant of the 1842 breakup of 3D/Biela ( Table 2).

\subsection{The Andromedid Meteor Showers}

When the breakup and subsequent loss of comet 3D/Biela was followed by spectacular meteor storms radiating from Andromeda on 1872 November 27 and again in 1885 (Nogami 1995), it was surmised that Earth had encountered the debris that was left from the comet (e.g., Newton 1894; Schulhof 1894; Lynn 1905; Olivier 1925).

According to Jenniskens (1995), the zenith hourly rate peaked at $\mathrm{ZHR}=7400 \pm 500 \mathrm{hr}^{-1}$ in 1872 and $6400 \pm 600 \mathrm{hr}^{-1}$ in 1885 , with other less well-observed but strong showers in 1892 $\left(\mathrm{ZHR} \sim 800 \mathrm{hr}^{-1}\right), 1899\left(\mathrm{ZHR}=150 \mathrm{hr}^{-1}\right)$, and $1940(\mathrm{HR} \sim$ $30 \mathrm{hr}^{-1}$ ) (see Table 3 of Jenniskens 2006). Others list rates uncorrected (or corrected differently) for radiant altitude, sky limiting magnitude, and observer perception, results of which differ by factors of 2-5 due to changing observing conditions from one return to the next. For example, Fisher (1926) cites hourly rates of 3000 (1872), 15,000 (1885), 6000 (1892), and 150 (1899). J. Trigo-Rodríguez pointed out that Catalan astronomer Comas Solà (1939) also observed the 1885 storm, reporting 4000 meteors $\mathrm{hr}^{-1}$ at maximum, which after correction is consistent with the ZHR values given by Jenniskens (1995), he concluded.

No similarly intense storms of Andromedids had been seen before, although accounts of Andromedid showers (rates up to a few hundred per hour) are known from 1741, 1798, 1830, 1838 , and 1847 , coincident with the return of the parent comet (Jenniskens 2006). These showers were distinctly different in appearance. Herrick (1863) described the relatively weak shower of Andromedids in the evenings of 1838 December 6 and 7 as "many large and splendid fireballs. . attended with trains" (Kronk 1988). Hawkins et al. (1959) spoke of two different Andromedid showers: those from the period 1741-1847 (all bright meteors?) and those from 1850-1899 (predominantly faint meteors).

Finally, there is a record of low-level activity from the Andromedids detected 100 years after the breakup of the comet (Dole 1924; Hawkins et al. 1959). Hawkins et al. (1959) identified Andromedids among photographed meteors captured by Harvard SuperSchmidt cameras in 1950-1955, deducing a visible rate of $0.2 \mathrm{hr}^{-1}$. By that time, the center of the stream had moved away from Earth orbit to a distance of about $0.04 \mathrm{AU}$ (Babadzhanov et al. 1991), stretching between November 2 and 22, with a mean node at about November 14. Today, the Andromedids are known from occasional fireballs centered on

TABLE 2

Parent Body and Associated Meteoroid Stream Following the 1842/1843 Breakup

\begin{tabular}{|c|c|c|c|c|c|c|c|}
\hline Object & Epoch & $\begin{array}{c}a \\
(\mathrm{AU})\end{array}$ & $\begin{array}{c}q \\
(\mathrm{AU})\end{array}$ & $\begin{array}{c}i \\
(\mathrm{deg})\end{array}$ & $\begin{array}{c}\text { Node (J2000.0) } \\
\quad(\mathrm{deg})\end{array}$ & $\begin{array}{c}\Pi \\
(\operatorname{deg})\end{array}$ & $\begin{array}{c}\text { Mass } \\
\left(10^{10} \mathrm{~kg}\right)\end{array}$ \\
\hline 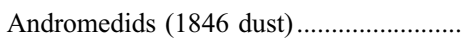 & 1872 & $\ldots$ & $\ldots$ & $\ldots$ & $\ldots$ & $\ldots$ & 32 \\
\hline Andromedids (1952 annual) .......................... & 1952 Nov & 2.90 & 0.777 & 7.5 & 225.5 & 108.2 & $<160$ \\
\hline 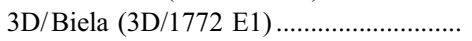 & 1772 Feb 21.0 & 3.613 & 0.990 & 17.06 & 260.94 & 114.28 & 1500 \\
\hline 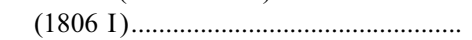 & 1805 Dec 25.0 & 3.570 & 0.907 & 13.59 & 254.08 & 112.15 & 210 \\
\hline 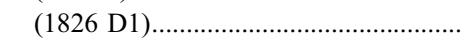 & 1826 Mar 30.0 & 3.561 & 0.902 & 13.56 & 253.98 & 112.24 & 1500 \\
\hline 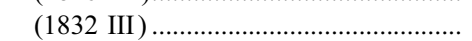 & 1832 Dec 3.0 & 3.519 & 0.879 & 13.22 & 250.67 & 112.33 & 530 \\
\hline 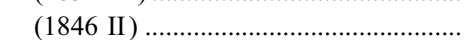 & 1846 Jan 24.0 & 3.518 & 0.856 & 12.58 & 248.14 & 111.20 & $930^{\mathrm{c}}$ \\
\hline (1852 III) & 1852 Sep 29.0 & 3.525 & 0.861 & 12.55 & 248.01 & 111.20 & $590^{\mathrm{c}}$ \\
\hline 3D/Biela-A ${ }^{\mathrm{a}}$ & 1951 Dec 20.0 & 3.505 & 0.819 & 9.39 & 227.17 & 108.94 & $\ldots$ \\
\hline 3D/Biela-A ${ }^{\mathrm{b}}$ & 1951 Dec 20.0 & 3.500 & 0.814 & 9.28 & 227.46 & 109.25 & $\begin{array}{l}\cdots \\
\cdots\end{array}$ \\
\hline $3 \mathrm{D} /$ Biela- $^{\mathrm{a}}$. & 2004 Jun 4.0 & 3.490 & 0.798 & 7.89 & 213.75 & 108.14 & $\ldots$ \\
\hline $3 \mathrm{D} /$ Biela- $\mathrm{A}^{\mathrm{b}}$. & 2004 Jun 4.0 & 3.523 & 0.813 & 7.99 & 201.57 & 109.23 & $\cdots$ \\
\hline 3D/Biela-A ${ }^{\mathrm{a}}$ & 2010 Dec 30.0 & 3.491 & 0.798 & 8.18 & 200.55 & 107.34 & $\cdots$ \\
\hline 3D/Biela-A ${ }^{\mathrm{b}}$ & 2010 Dec 30.0 & 3.493 & 0.795 & 7.93 & 195.22 & 108.49 & $\ldots$ \\
\hline 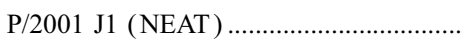 & 2001 May 7.0 & 3.877 & 0.937 & 10.16 & 200.80 & 111.82 & 60 \\
\hline
\end{tabular}

\footnotetext{
${ }^{\text {a }}$ Future orbit of fragment 3D-A according to K. Kinoshita (see footnote 3).

${ }^{b}$ Same for forward integration of the 1832 Biela orbit using JPL/Horizons.

${ }^{\mathrm{c}}$ Primary component.
} 


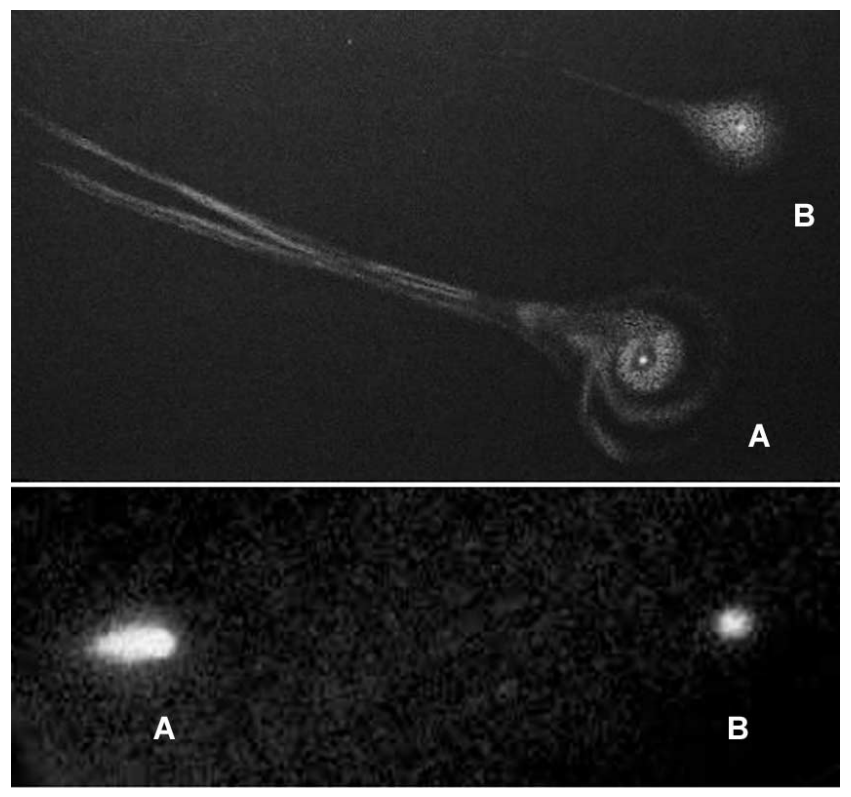

FIG. 1.-Comet 3D/Biela in February of 1846 (top) in a drawing by E. Weiss (Vienna), as reproduced from Bilderatlas der Sternenwerlt (1888), and on 1852 September 25 (bottom) in a drawing by O. W. Struve at Pulkovo Observatory. North is up, and west is to the right. The components A and B are marked.

November 1. The level of visual activity in 1979 was ZHR < $0.5 \mathrm{hr}^{-1}$ (Wood 1980). We estimate that to within an order of magnitude the mass of the annual component is about $M<$ $160 \times 10^{10} \mathrm{~kg}$ for particles in the range $10^{-6} \mathrm{~g}$ to $10 \mathrm{~kg}$, if the magnitude distribution is $\chi=2.5$, a typical value for annual shower particles that have been exposed to collisions with interplanetary meteoroids (Trigo-Rodríguez et al. 2005; Jenniskens 2006).

\subsection{The Earlier Efforts to Model the Andromedid Storms}

The early efforts to explain the Andromedid showers were mostly concerned with bridging the gap between the comet orbit and Earth. Several authors suggested that the showers were due to different fragments of the comet that had been ejected with some speed in earlier years. Newton (1894) pointed out that the nodes of 1798,1838 , and $1846(\Omega \sim 257.7)$ were that of the comet orbit in 1772, while the nodes of the 1867,1872 , and 1885 returns $(\Omega \sim 247.7)$ correspond to the orbit in 1846. Schulhof (1894) proposed that the Andromedids of 1798 and 1838 were from a fragment detached in 1772 that gradually moved ahead of the comet and had gained 4 months on the comet itself by 1798 and 7 months by 1838 . As a variation on that theme, Brédichin (1893) identified the 1877,1885 , and 1892 showers as due to "nuclear ejections" from 1846 with high speeds of 292, 342, and $279 \mathrm{~m} \mathrm{~s}^{-1}$, respectively.

We now know that meteoroids and comet orbits evolve independently under the influence of planetary perturbations. This evolution was investigated by Makhmudov (1982) and Katasev \& Kulikova (1981a, 1981b). It was found that perturbations moved the node outside of Earth orbit after the return of 1892, when the Andromedids were still strong.

After ejection during each return, the cloud of dust spreads in a thin dust trail after one orbit due to differences in orbital period of the meteoroids caused by ejection velocity and solar radiation pressure. In a paper written in Russian, Reznikov (1982) first identified the dust trails responsible for the 1872 and 1885 storms as those of 1839 and 1846 (1872) and 1846 and 1852 (1885). He used the simplified assumption of ejection of dust at perihelion, in the forward direction, which has since proven useful for predicting Leonid meteor storms.

\section{A DUST TRAIL MODEL}

We applied a new model for the formation and evolution of comet dust trails, developed by Vaubaillon (2004) and Vaubaillon et al. (2005), to reexamine the cause of the Andromedid storms. The model evolves a cloud of dust grains ejected according to the Crifo model for comet dust ejection (Crifo \& Rodinov 1997), which is a slight adaptation of Whipple's comet ejection model (Whipple 1951), by calculating the planetary perturbations on 50,000 particles $(10,000$ each in five mass bins). The meteoroids are ejected in a uniform manner on the sunlit hemisphere in directions at angles in the cometary plane $(\alpha)$ and out of the plane of the cometary orbit $(\beta)$. The distribution of $\alpha$ is uniform. That of $\beta$ is such that:

$$
\begin{gathered}
P=\operatorname{ran}(i), \text { over the range }[0,1], \\
\theta=\operatorname{acos}[(1-2) P], \text { over the range }[0, \pi], \\
\beta=\pi / 2-\theta, \text { over the range }[-\pi / 2, \pi / 2] .
\end{gathered}
$$

Early results were reported in Jenniskens (2006). Here we investigate whether the observed Andromedid storms were due to dust released (1) during the initial fragmentation at aphelion in 1842/1843, (2) during the progressive disintegration of comet fragment A (and B?) in the returns of 1846 and 1852, or (3) during normal activity from the evaporation of ices at perihelion by fragments A and B during the 1846 and 1852 returns.

\subsection{Dust Ejection at Aphelion}

Dust ejected at aphelion has the peculiar property that the kinetic energy of the meteoroids changes the perihelion distance, rather than the orbital period, as a result of which the meteoroids are not quickly dispersed. Neither the exact time of the fragmentation event during the $1842 / 1843$ period nor the conditions of dust ejection are important for the overall features of the subsequent dust evolution. We developed a model in which dust was ejected at aphelion in December of 1842 in isotropic emission at low $\left(\mathrm{a} \mathrm{few} \mathrm{m} \mathrm{s}{ }^{-1}\right.$ ) ejection speed. Note that according to Marsden \& Sekanina (1971), the secondary left the primary with a relative speed toward the Sun of about $1 \mathrm{~m} \mathrm{~s}^{-1}$.

We find that the dust does not evolve far from the comet itself and did not approach Earth during the storms of 1872 and 1885 (Fig. 2). Because ejection occurs at aphelion, the orbital period differences are less than would occur for ejection at perihelion with the same speed. The cloud stays mostly together and was found to have detached from the position of the comet in the 1885 return.

\subsection{Dust Ejected along the Comet Orbit in Normal Water Vapor Drag}

The orbit of comet 3D/Biela can be reliably calculated back some time before the first sighting in 1772. Marsden \& Sekanina (1971) showed that the comet (even including the main mass in the 1846 and 1852 returns) behaved in a well-defined manner between 1772 and 1852 . We used this solution to integrate the comet orbit back to 1703 . 

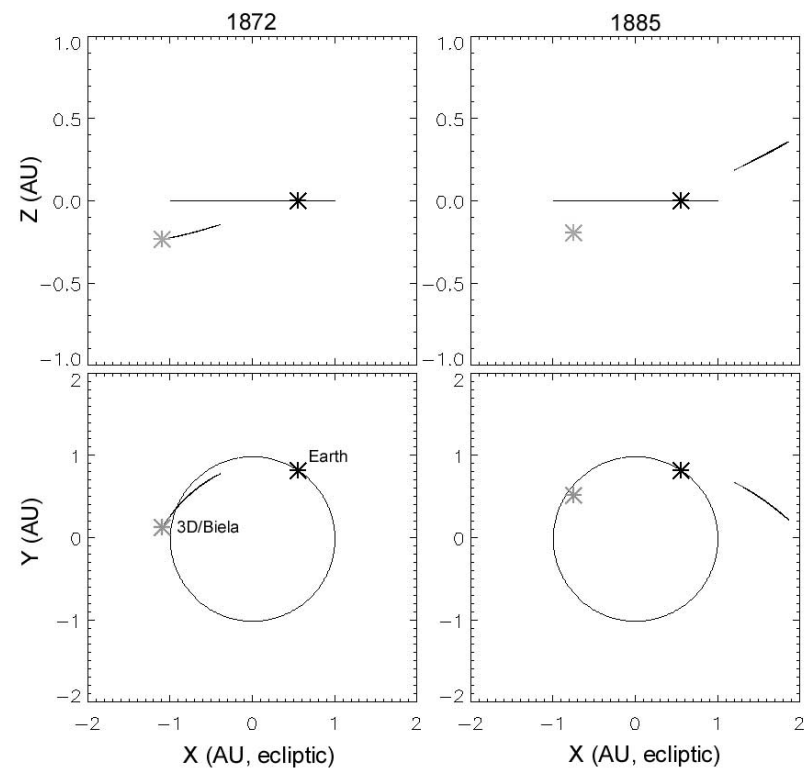

FIG. 2.-Position of dust ejected by comet 3D/Biela at aphelion in December of 1842 at the time of the meteor storms of 1872 and 1885 .

Starting from this 1703 orbit, we calculated the position of the dust trails at Earth orbit that evolved from normal Whipple-type ejection of meteoroids (based on the model by Crifo \& Rodinov 1997) during each of the returns between 1703 and 1866 (24 revolutions). For purposes of evaluating the relative dust density, we assumed that the comet was similarly active in each return, based on the similar level of activity during the few observed returns. Each return created a new dust trail, reflecting how the comet itself was perturbed. We evaluated where each trail was located near Earth's orbit at the time of Earth passage by the stream in November/December in the following years.

Figure 3 shows the position of the node of dust particles in the ecliptic plane near Earth orbit in the years when meteoroids were close to Earth in the 2 weeks before and after the encounter. Figure 4 shows the position of these dust trails at the time of the meteor storms. Each dust trail is shown as a distribution of nodes for individual meteoroid orbits at the time of encounter. The density of points reflects the density of meteoroids in the trail at the time of encounter. In Figure 4 the trails nearest to Earth orbit are marked with the year of the comet's return when the dust was released. Table 3 summarizes the statistical properties of each dust trail encounter, in terms of the nearest distance to the trail center in the heliocentric radial direction $(\Delta r)$, the initial difference in orbital period (in terms of $\Delta a$ ), and the dust density compared to that in the unperturbed one-revolution dust trail $\left(f_{m}\right)$.

As long as ejection speeds (or the relative speed between comet fragments and meteoroids) are low, the position of the trail is determined mostly by planetary perturbations and the time of ejection of the grains along the comet orbit. Small discrepancies in the trail position between observed and calculated positions were observed for the Leonid dust trails (from comet 55P/Tempel-Tuttle), where the trails were calculated systematically +0.00077 AU too far out from the Sun (Jenniskens 2006).

We find that the 1872 and 1885 storms were caused by dust from the 1846 breakup, with only minor contributions from dust ejected in 1839 and 1852, respectively (Fig. 3). Again, the dust distribution appears to be calculated slightly further out from the Sun than observed.

Figure 4 shows the detailed distribution of dust from the 1846 dust trail. A theoretical activity profile was derived by counting

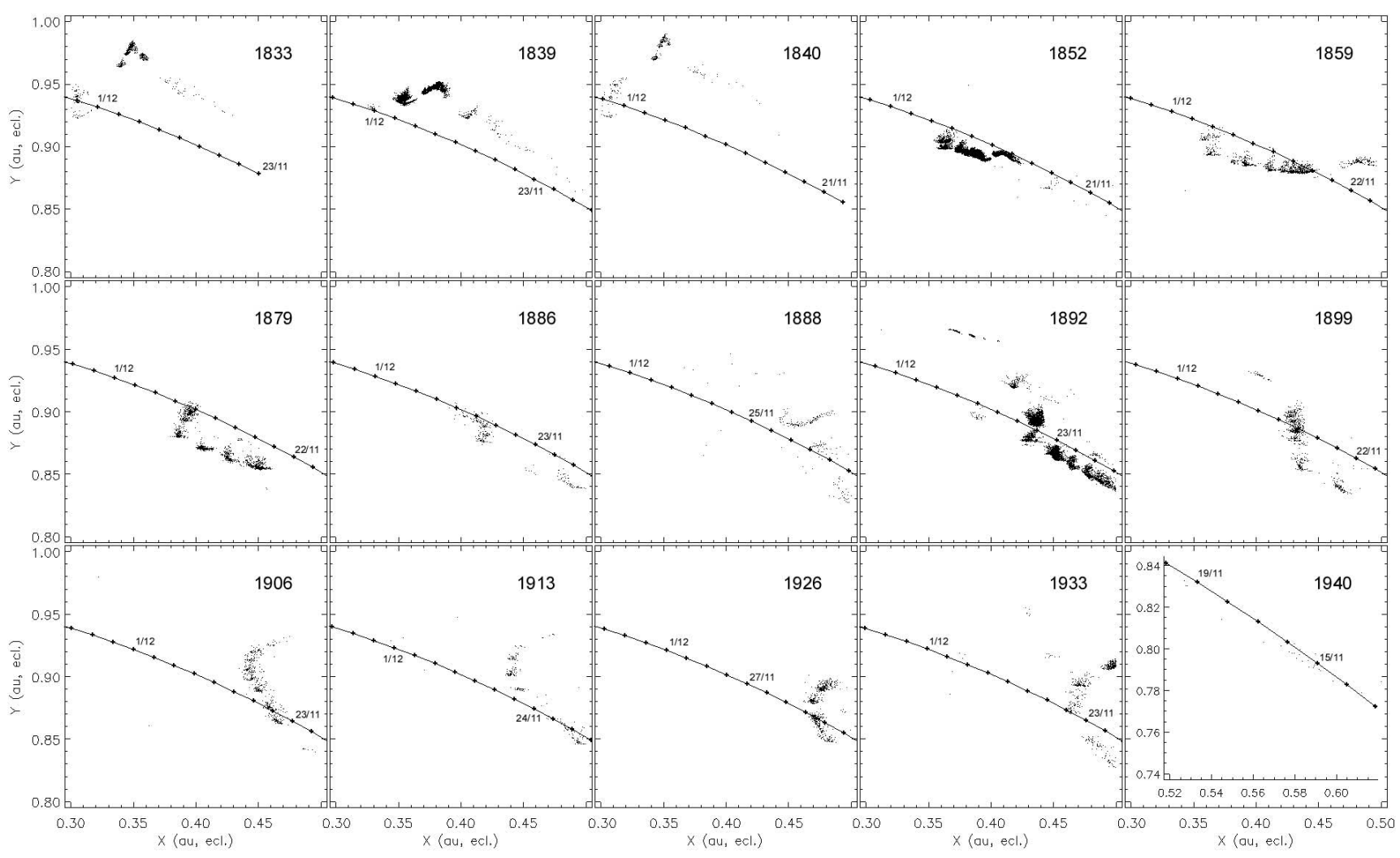

Fig. 3.-Position of comet 3D/Biela's dust trails near Earth orbit at times when meteoroids pass Earth orbit. The ecliptic plane is shown, with each small dot marking the node of a meteoroid orbit ejected during previous returns. 


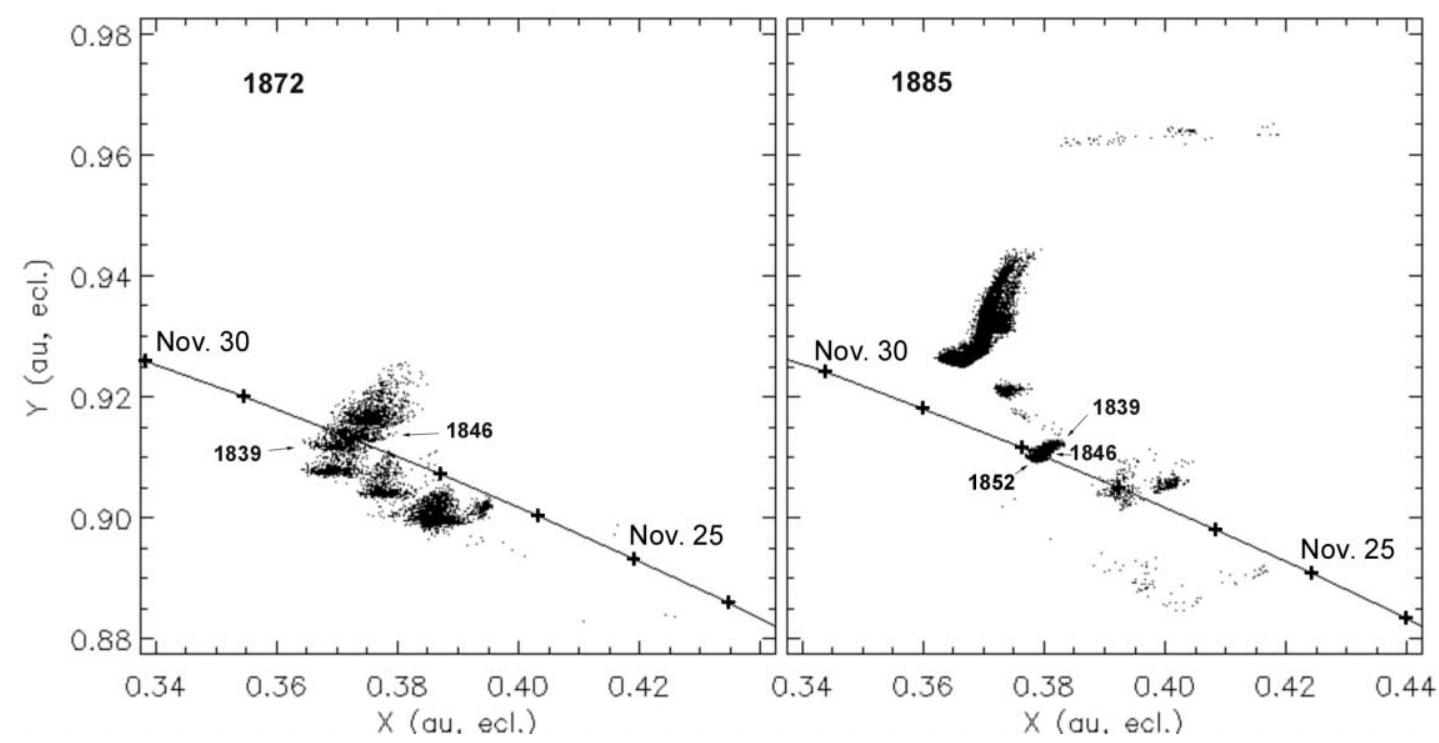

Fig. 4.-Same as Fig. 3, but during the meteor storms of 1872 and 1885. The relevant dust trails are marked by their year of origin.

(in small intervals of solar longitude) the density of meteoroids in the model with nodes at heliocentric distances between +0.0000 and +0.0015 AU outside Earth orbit, arriving at Earth orbit over a period of 1 week before and after the time of the storm. The resulting rate profile is matched to the observed rate of meteors (from Jenniskens 1995) in Figure 5, equivalent to each particle in the model corresponding to a $\mathrm{ZHR}=50 \mathrm{hr}^{-1}$.

The calculated profile for the 1846 dust trail crossing in 1872 is wider than observed (Fig. 5, circles). The excess of meteoroids at high solar longitude represents particles that were ejected out of the plane of the comet orbit with angles $\beta>30^{\circ}$ on the southern hemisphere (prior to the peak) when the comet was located inside Earth orbit (Fig. 6). Otherwise, these particles were ejected with normal ejection speed $\left(\sim 20 \mathrm{~m} \mathrm{~s}^{-1}\right)$ and at reasonable heliocentric distances. Hence, the lack of particles in this regime implies that the comet lost dust preferentially in the plane of the comet orbit.

The situation in 1885 is different (Fig. 6). All observed meteoroids represent particles that were ejected in 1846 with a small $\beta<40^{\circ}$, during and shortly after passing perihelion. Particles ejected just before perihelion were ejected with a high $\beta \sim 30^{\circ}-$ $60^{\circ}$ away from the Sun-comet direction, while particles ejected after perihelion were ejected with a similarly high angle away from the Sun-comet direction. In this case the width of the observed activity curve is in good agreement with the calculated distribution. This suggests that some particles were indeed ejected at relatively high Sun-comet angles in the plane of the comet orbit. The calculated maximum in the activity profile, however, falls $0.02^{\circ}$ in solar longitude after the observed peak. Given that the distribution of nodes is skewed to lower nodes further away from Earth orbit, this could imply that Earth crossed the dust trail at a distance of $+0.0010 \mathrm{AU}$ from the calculated position. Particles from the 1852 dust trail contribute some activity before the peak (Fig. 5).

We find that all other strong meteor outbursts reported in the literature, those of 1886 ("stars fell like rain"), 1892 (HR 6000), and 1899 (HR 150), can be identified with Earth crossing the 1852 ejecta. To obtain high rates in 1892 (and no shower in 1879), the dust trail must again have been slightly more toward the Sun than calculated. A difference of $+0.00075 \mathrm{AU}$ is not sufficient $(\mathrm{ZHR} \sim 50$ ), but about twice that value is implied.
In contrast, results for the dust trail of 1899 are less sensitive to the exact location at which the dust trail was crossed. Based on the calculated time of the crossing, the peak of the shower appears to have been observed by Father Algué of Georgetown College at an apparent rate of 48 mostly faint meteors in 5 minutes (Hagen 1892). Again, a good fit to the 1899 dust trail data is obtained for one particle being equal to $\mathrm{ZHR}=50$. This suggests that the amount of dust ejected in 1846 was similar (to within a factor of 2) to the dust ejected in the 1852 return.

The last to witness Earth's passage through a dust trail of what is now the " $\mathrm{D}$ " comet 3D/Biela was experienced AMS observer R. M. Dole of Cape Elizabeth, Maine (Prentice 1941). In the evening of 1940 November 15, when Europe was occupied with the war, Dole observed a peak of faint Andromedid meteors of $30 \mathrm{hr}^{-1}$, the cause of which has now been identified as the 1852 dust trail wandering in Earth's path. The meteoroids that year were dispersed over a relatively wide region in solar longitude (Fig. 3).

Other dust trails (those of 1792, 1799, 1826, and 1832) would only have caused weak showers in 1833, 1839, 1840, 1859, 1888, and 1926. None of these weak showers have been reported. On closer inspection, however, none were very favorable for detection. The most likely shower to have been seen is that caused by the 1832 dust trail in 1926, when the Moon was unfavorable. A similarly rich shower from the 1846 dust trail in 1933 was not reported.

We were not able to identify the meteor outbursts reported in 1741, 1798, 1830, 1838, and 1847, when Andromedids peaked at rates of $100-400 \mathrm{hr}^{-1}$ and appeared to have been brighter on average (Benzenberg \& Brandes 1800, pp. 80-81). It is likely that these outbursts resulted from dust generated prior to 1703 .

\section{FRAGMENTING VERSUS SUBLIMATING COMETS}

\subsection{The Two Streams: The Meteoroid Size Distribution Index}

Our model shows that the storms of 1872 and 1885 should have contained large particles, if they were present in the stream. Instead, these showers (and all other showers seen after 1846 and now identified with crossings of the 1846 and 1852 dust trails) were all rich in faint meteors, with $\chi=3.6, \chi$ being the number 
TABLE 3

Encounters of Earth with 1703-1866 3D/Biela Dust Trails

\begin{tabular}{|c|c|c|c|c|c|c|c|c|c|c|c|c|c|}
\hline Year & Trail & $\begin{array}{c}\Delta r \\
(\mathrm{AU})\end{array}$ & $\begin{array}{l}\Delta a_{0} \\
(\mathrm{AU})\end{array}$ & $f_{m}$ & $\begin{array}{c}\text { Predicted Shower } \\
\lambda_{0}(\mathrm{~J} 2000.0) \\
(\mathrm{deg})\end{array}$ & $\begin{array}{c}\text { Shower } \\
\lambda_{0}(\mathrm{~J} 2000.0) \\
(\mathrm{deg})\end{array}$ & $\begin{array}{l}\text { Time } \\
\text { (UT) }\end{array}$ & $\begin{array}{l}\text { Radiant R.A. } \\
\text { (J2000.0) } \\
\text { (deg) }\end{array}$ & $\begin{array}{l}\text { Radiant Decl. } \\
\text { (J2000.0) } \\
\text { (deg) }\end{array}$ & $\begin{array}{l}\text { Predicted ZHR } \\
\qquad\left(\mathrm{hr}^{-1}\right)\end{array}$ & $\begin{array}{c}\text { Observed ZHR } \\
\quad\left(\mathrm{hr}^{-1}\right)\end{array}$ & $\begin{array}{c}\text { Moon Phase } \\
(\%)\end{array}$ & Moon Position \\
\hline $1829 \ldots \ldots \ldots$ & 1758 & -0.00093 & +0.1329 & 0.046 & 257.637 & $\ldots$ & Dec 7 13:33 & No report & No report & $\sim 50$ & $\ldots$ & 88 & Cet \\
\hline $1833 \ldots \ldots$. & 1799 & -0.00052 & +0.0807 & 0.063 & 251.917 & $\ldots$ & Dec 1 22:58 & No report & No report & 50 & $\ldots$ & 76 & $\mathrm{Cnc}$ \\
\hline $1839 \ldots \ldots$. & 1832 & +0.0020 & +0.1461 & 0.109 & 250.547 & $\ldots$ & Dec 1 03:29 & No report & No report & 200 & $\ldots$ & 21 & Vir \\
\hline $1840 \ldots \ldots \ldots$ & 1799 & +0.00436 & +0.0838 & 0.059 & 251.842 & $\ldots$ & Dec 1 16:15 & No report & No report & $<50$ & $\ldots$ & 42 & $\ldots$ \\
\hline $1859 \ldots \ldots \ldots$ & 1799 & -0.00442 & +0.0220 & 0.136 & 243.486 & $\ldots$ & Nov 24 07:20 & No report & No report & 150 & $\ldots$ & 0 & Sco \\
\hline \multirow[t]{3}{*}{$1872 \ldots \ldots \ldots$} & 1839 & -0.00164 & +0.0199 & 0.184 & 247.909 & 247.762 & Nov 28 00:12 & 27.3 & +43.8 & 500 & 7400 & 10 & Vir \\
\hline & 1846 & +0.00119 & +0.0222 & 0.249 & 247.749 & $\ldots$ & Nov 27 20:24 & $\ldots$ & $\ldots$ & 3700 & $\ldots$ & & $\ldots$ \\
\hline & 1852 & +0.00404 & +0.0268 & 0.287 & 247.701 & $\ldots$ & Nov 27 19:16 & $\ldots$ & $\ldots$ & $<50$ & $\ldots$ & $\ldots$ & $\ldots$ \\
\hline $1872 \ldots \ldots \ldots$ & 1826 & -0.00605 & +0.0175 & 0.264 & 247.323 & $\ldots$ & Nov 27 10:18 & No report & No report & 75 & $\ldots$ & 13 & Vir \\
\hline $1879 \ldots \ldots . .$. & 1852 & -0.00514 & +0.0505 & 0.157 & 246.279 & $\ldots$ & Nov 27 04:38 & No report & No report & 60 & $\ldots$ & 98 & Ari \\
\hline \multirow[t]{2}{*}{$1885 \ldots \ldots}$. & 1846 & +0.00032 & -0.0060 & 0.285 & 247.385 & 247.389 & Nov 27 19:44 & 26.2 & +45.3 & 10500 & 6400 & 65 & Leo \\
\hline & 1852 & -0.00070 & -0.0057 & 0.211 & 247.399 & .. & Nov 27 20:04 & & $\cdots$ & 450 & $\ldots$ & r. & .. \\
\hline $1886 \ldots \ldots$. & 1852 & -0.00425 & +0.069 & 0.091 & 244.782 & 245.0 & Nov 25 12:08 & $\begin{array}{l}\text { Stars fell like } \\
\text { rain in China }\end{array}$ & $\begin{array}{l}\text { Stars fell like } \\
\text { rain in China }\end{array}$ & $<50$ & ? & 0 & Sco \\
\hline $1888 \ldots \ldots \ldots$ & 1792 & +0.01940 & 0.0562 & 0.191 & 242.194 & $\ldots$ & Nov 22 11:12 & No report & No report & $<50$ & $\ldots$ & 98 & $\ldots$ \\
\hline $1892 \ldots \ldots \ldots$ & 1852 & +0.00428 & +0.0211 & 0.214 & 243.880 & 243.809 & Nov 24 03:43 & 27.2 & +40.8 & $50-4500$ & $\sim 800$ & 18 & Sgr \\
\hline \multirow[t]{2}{*}{$1899 \ldots \ldots}$. & 1846 & -0.00853 & +0.0386 & 0.090 & 243.843 & 243.7 & Nov 24 22:00 & 25.2 & +43.0 & $<50$ & $\mathrm{HR} \sim 150$ & 55 & Leo \\
\hline & 1852 & -0.00231 & +0.0394 & 0.189 & 243.983 & $\ldots$ & Nov 25 01:18 & $\ldots$ & $\ldots$ & 200 & $\ldots$ & $\ldots$ & $\ldots$ \\
\hline $1906 \ldots \ldots \ldots$ & 1846 & +0.00198 & +0.0546 & 0.102 & 242.422 & $\ldots$ & Nov 24 07:17 & No report & No report & $<50$ & $\ldots$ & 56 & Aqr \\
\hline $1913 \ldots \ldots .$. & 1846 & -0.00152 & +0.0688 & 0.059 & 240.638 & $\ldots$ & Nov 22 08:03 & No report & No report & 75 & $\ldots$ & 38 & Leo \\
\hline $1926 \ldots \ldots \ldots$ & 1832 & +0.01189 & 0.0443 & 0.513 & 241.500 & $\ldots$ & Nov 23 12:33 & No report & No report & 290 & $\ldots$ & 84 & $\ldots$ \\
\hline $1933 \ldots \ldots . .$. & 1846 & +0.00456 & 0.0423 & 0.067 & 241.955 & $\ldots$ & Nov 23 18:18 & No report & No report & 300 & $\cdots$ & 41 & $\ldots$ \\
\hline $1940 \ldots \ldots \ldots$ & 1852 & -0.00029 & +0.0722 & 0.043 & 234.174 & 234.2 & Nov 15 20:30 & Peak of faint meteors & Peak of faint meteors & $<50$ & $\mathrm{HR} \sim 30$ & 99 & Tau \\
\hline
\end{tabular}

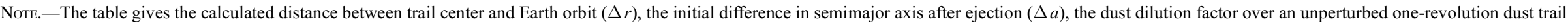

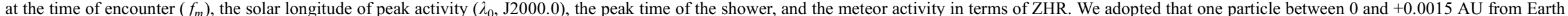
orbit in the model means $\mathrm{ZHR}=50 \mathrm{hr}^{-1}$. 

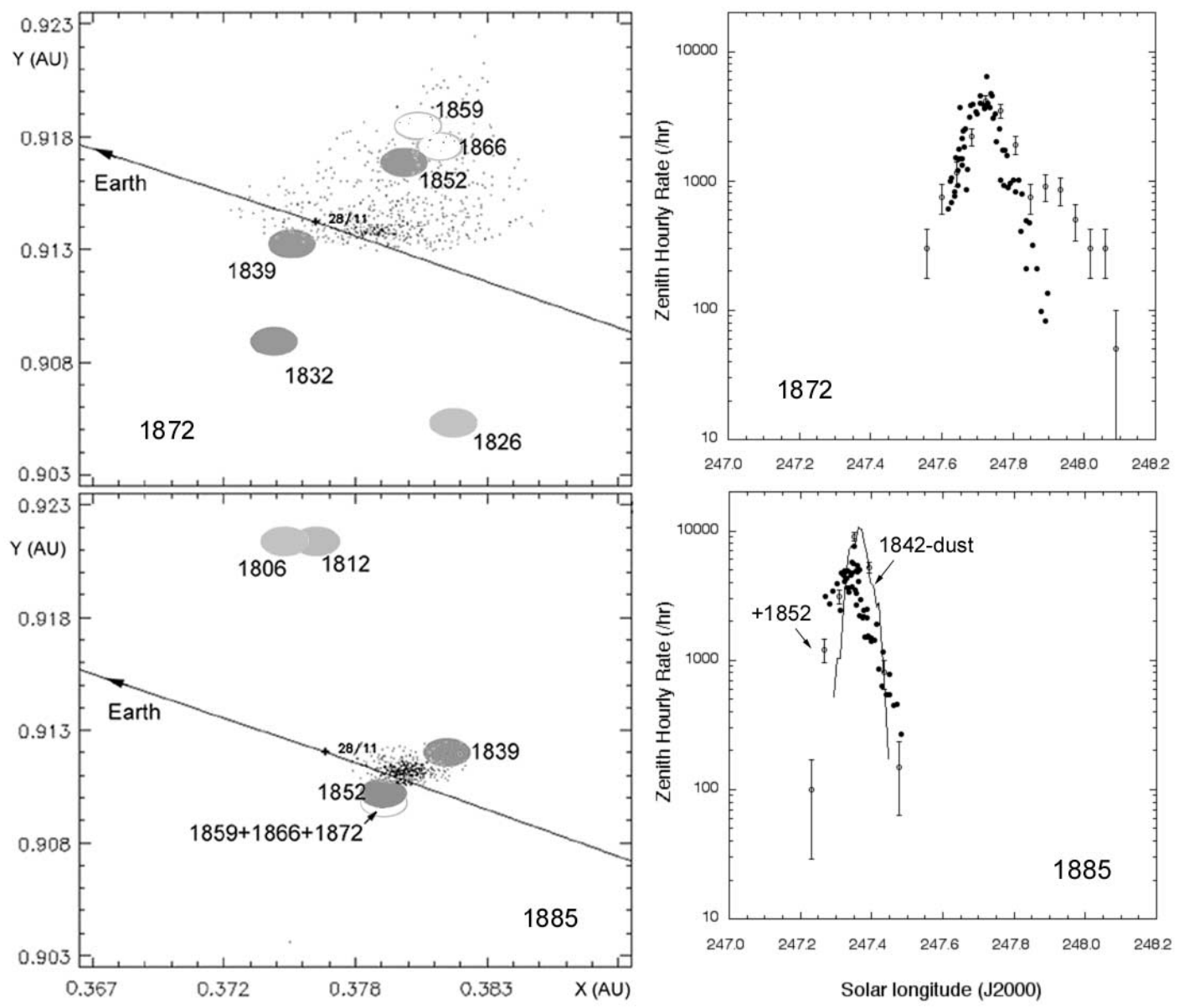

FIG. 5. - Extracted activity curve from the distribution of nodes in Fig. 4 compared to the observed ZHR during the 1872 and 1885 Andromedid storms. The distribution of nodes in the dominant 1846 dust trail is shown on the left (detail of Fig. 4).

ratio of meteors in neighboring magnitude intervals (Jenniskens 1995).

Other dust trails evolved from Whipple-type ejection in Halleytype comets (Leonids, Perseids) have a size distribution index that is much more flat, with $\chi \sim 2.15$. This low value of $\chi$ is representative of a collisional cascade, whereby particles are typically broken by collisions with other grains of similar mass (Dohnanyi 1969). When the distribution is collisionally relaxed into having equal cross section per log mass interval, $\chi=1.85$. Indeed, descriptions of the early Andromedid showers in the years 1741-1838 are more consistent with such a low value of $\chi$.

The 1846 and 1852 Andromedids, on the other hand, broke into a size distribution with most mass at smaller sizes, past having equal distribution of mass per log mass interval $(\chi=2.5)$, which would result as a product of catastrophic fragmentation in collisions with other meteoroids of the zodiacal cloud (Fujiwara et al. 1989; Jenniskens 2006). This implies that large grains were efficiently lost from the population by another means than collisions. Exposure to solar radiation may have broken the larger grains shortly after ejection. This did not happen to early Andromedids as much, suggesting the grains released during 1846 and 1852 initially contained more volatiles. This argues against option 3 but in favor of option 1 or 2 .

The high-magnitude distribution index does not immediately argue in favor of fragmentation. The dust generated from comet D/1993 F2 (Shoemaker-Levy 9) when it was disrupted at Jupiter had a distribution index more typical of $\chi=1.85$ (Hahn \& Rettig
2000). Also, the Draconid storms of 1933 and 1946 were rich in faint meteors and very fragile, but they are not thought to have been created in a breakup of parent comet 21P/Giacobini-Zinner (Beech 1986).

\subsection{The Mass in the Stream}

Our earlier result for the mass in the Andromedid stream of $3.3 \times 10^{10} \mathrm{~kg}$ (following the method described in Jenniskens 1995 ) assumed that only $1 / 30$ th of its orbit was filled with meteoroids at the density encountered during the 1872 and 1885 storms. Instead, we now find that much of the mass is located closer to the comet orbit. The model provides a calculated distribution of dust. In our model of the 1846 dust trail in 1872,627 particles out of a total of 50,000 are within the 13 day period centered on the time of encounter.

From this, and following the same method as before, we calculate a total dust trail mass for particles in the range of onemillionth $\left(10^{-6}\right)$ to $100 \mathrm{~g}$ of $32 \times 10^{10} \mathrm{~kg}^{\mathrm{g}}$ orbit $^{-1}$ (for $\chi=3.6$ ), an order of magnitude larger than our previous estimate. If the mass distribution index is $\chi=2.5$ instead, then a total mass of $51 \times 10^{10} \mathrm{~kg} \mathrm{orbit}^{-1}$ results.

\subsection{Nongravitational Forces}

The amount of dust lost during the 1846 and 1852 returns was about a factor of 10 larger than in a normal return of the comet. From the nongravitational changes in the orbit of the comet in the years of normal activity (1772-1832), some of the largest 


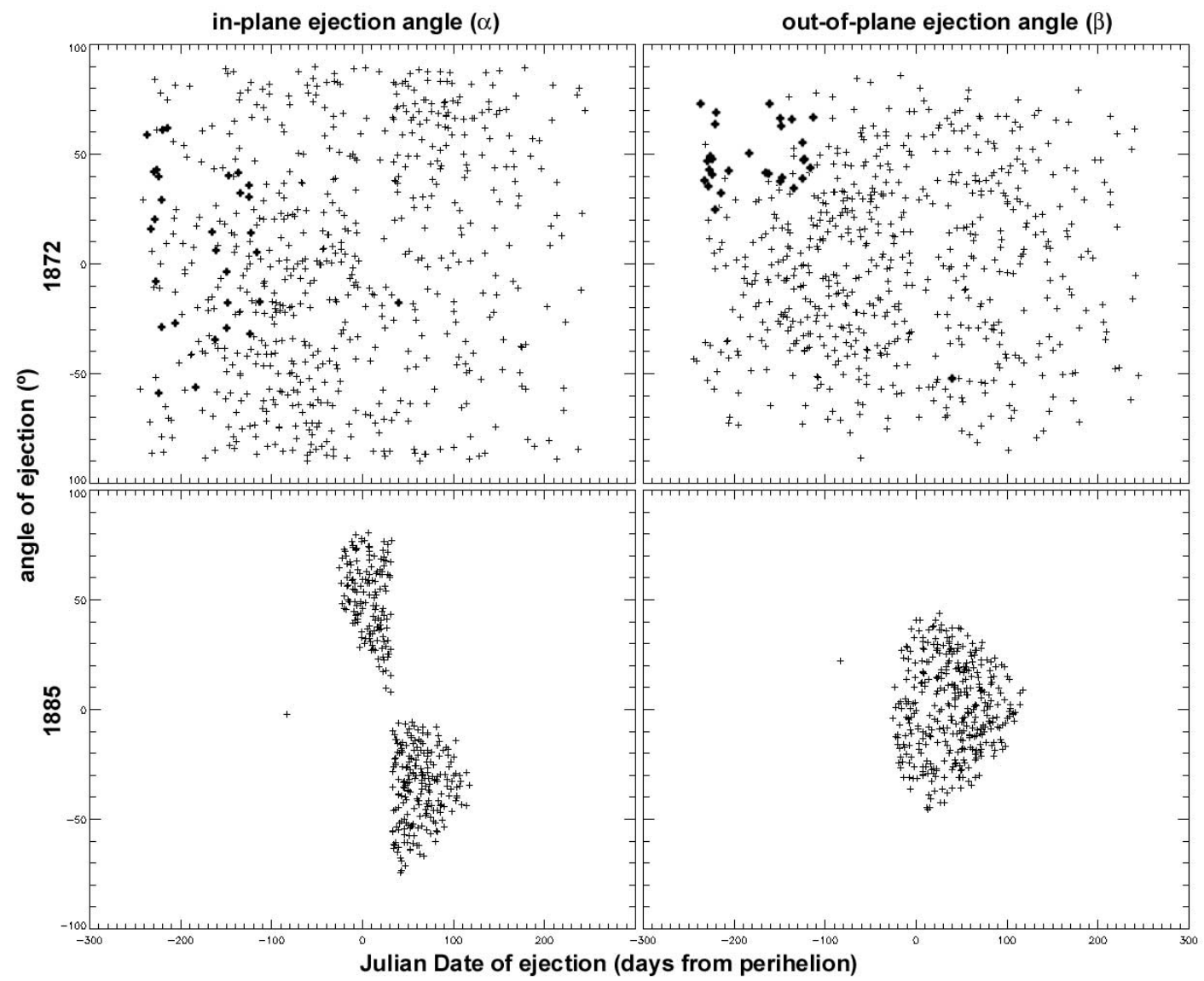

FIG. 6.-Distribution of ejection angles in and out of the plane of the comet orbit for dust encountered during the meteor storms of 1872 and 1885 (dust with nodes between +0.0000 and +0.0015 AU from Earth's orbit). Thick points mark dust grains found at high solar longitude in the upper wing of the activity curve profile $(X<0.371 \mathrm{AU})$.

on record, Whipple (1951) calculated a loss rate of all matter to be $\Delta M / M=0.0047$ orbit $^{-1}$, which translates to about $4.8 \times$ $10^{10} \mathrm{~kg}$ orbit $^{-1}$ for his adopted upper limit to the size of $3 \mathrm{D} /$ Biela $(3.4 \mathrm{~km})$ and $0.94 \times 10^{10} \mathrm{~kg} \mathrm{orbit}^{-1}$ for the size of $2.52 \mathrm{~km}$ adopted by Hughes (2003). The diameter estimated by Jenniskens (2006) is in the middle, $D=3.0 \mathrm{~km}$, giving a mass loss of $1.6 \times 10^{10} \mathrm{~kg}$ orbit $^{-1}$. This is identical to the mass loss of $10^{9}-10^{11} \mathrm{~kg}$ orbit ${ }^{-1}$ of other Jupiter-family comets with observed comet dust trails (Sykes \& Walker 1992).

Marsden \& Sekanina (1971) calculated nongravitational parameters for the primary comet component in the nonbroken years $1806-1932$ of $10^{5} A_{1}=+2.78 \pm 0.10$ and $10^{6} A_{2}=-2.5037 \pm$ 0.0044 , while the same values for the orbits in 1832, 1846, and 1852 were $10^{5} A_{1}=+3.56 \pm 0.52$ and $10^{6} A_{2}=-2.5965 \pm$ 0.0061 . Hence, the nongravitational forces did not change by a factor of 10 after breakup.

This implies that the 10 times larger mass was lost in a process that did not impart a strong rocket effect on the comet itself. On the other hand, the particles did need to be accelerated to achieve the typical ejection speed of $20 \mathrm{~m} \mathrm{~s}^{-1}$ that follows from the dispersion of the dust in the activity profile. One way to achieve this is by having fragments crumble from the comet that then evaporate to eject the dust particles.

\subsection{On the Relative Contributions from Fragments $A$ and $B$}

Marsden \& Sekanina (1971) have a difference in node for the orbits of fragments A and B of only $0.0036^{\circ}$ in the return of 1846 and $0.0022^{\circ}$ in 1856 . That is too small to cause a noticeable dif- ference in the time of the shower peak for dust ejected by the primary or secondary component.

It was the primary that was observed to shed larger fragments during the return, and it is likely that most mass originated from the primary component. Marsden \& Sekanina found that the splitting resulted in a strong impulsive change in the secondary, presumably because it was less massive. The secondary had a nongravitational deceleration, comparable to or possibly larger than the secular acceleration of the primary.

\subsection{Will 3D/Biela Be Recovered As a Dormant Comet?}

The total mass of dust in the Andromedid stream is still much less than the mass of comet $3 \mathrm{D} /$ Biela (Table 1), and we suspect that more matter was lost at some other time. It is possible that the comet fell apart completely in the returns of 1859 or 1866. Unfortunately, the lack of dust trail crossings with the 1859 and 1866 dust trails prevents us from knowing whether the comet fell apart completely during these returns. The resulting dust trails would not have caused meteor storms on Earth. It is difficult to understand, however, why the complete disintegration of the comet took at least two orbits to commence and then happened unnoticed.

It is more likely that most of the mass loss occurred in the initial breakup. If the comet lost about half its mass in the 1842/1843 breakup, then its magnitude should have gone from +7.5 to +8.3 (Jenniskens 2006). That is not inconsistent with the magnitude estimates for the primary component during the 1846 and 1852 returns. Again, most of that mass was never in Earth's path. 
In that case, at least fragment A may have survived until the present day, now hiding as a dormant comet. If so, K. Kinoshita ${ }^{3}$ calculated a particularly good encounter in 2010, when the dormant comet is expected to pass Earth at only 0.13 AU on November 3.25, following a close encounter with Jupiter (0.79 AU) on 2009 March 13.5. His orbital elements for epoch 2010 December 30 are given in Table 2 .

\section{CONCLUSIONS}

All Andromedid storms and outbursts characterized by faint meteors after 1850 were due to either the 1846 or 1852 dust trails of comet 3D/Biela. The total mass in the dust trails is about a factor of 10 larger than that ejected during the normal activity of the comet. These grains crumbled efficiently, presumably because they contained a higher content of volatiles after release. The ongoing crumbling of the comet, instead of normal water vapor drag, is implicated.

\footnotetext{
${ }^{3}$ See the Comet Orbit Home Page at http://www 9.ocn.ne.jp/ comet/.
}

Andromedid outbursts prior to 1850, and the meteoroids encountered now in a weak annual component, appear to be richer in bright meteors, more typical of dust generated in normal water vapor drag. This dust was generated prior to 1703 , the start of our integrations.

The process of crumbling during the 1846 and 1852 returns did not account for much of the comet's mass loss during fragmentation. The Andromedid trails of 1846 and 1852 account for only about $5 \%$ of the comet's original mass. If the breakup of 3D/Biela produced an amount of dust comparable to the mass of the remaining fragment (as in other such breakups), then the main mass loss may have occurred at aphelion during the initial breakup. Unfortunately, that dust did not disperse sufficiently quickly along the comet orbit for it to have been seen at Earth in later years.

We thank NASA's Planetary Astronomy program for support of this work.

\section{REFERENCES}

Babadzhanov, P. B., Wu, Z., Williams, I. P., \& Hughes, D. W. 1991, MNRAS, 253, 69

Beech, M. 1986, AJ, 91, 159

Benzenberg, J. F., \& Brandes, H. W. 1800, Versuche die Entfernung, die Geschwindigkeit und die bahnen der Sternschnuppen zu bestimmen (Hamburg: Friedrich Perthes)

Brédichin, T. 1893, Astron. Nachr., 132, 191

Challis, J. 1846, MNRAS, 7, 90

Comas Solà, J. 1939, Astronomía (Barcelona: Ed. Ramón Sopena)

Crifo, J.-F., \& Rodinov, A. V. 1997, Icarus, 127, 319

Dohnanyi, J. S. 1969, J. Geophys. Res., 74, 2531

Dole, R. M. 1924, Popular Astron., 32, 389

Fisher, W. J. 1926, Proc. Natl. Acad. Sci., 12, 728

Fujiwara, A., Cerroni, P., Davis, D., Ryan, E., \& di Martino, M. 1989, in Asteroids II, ed. R. P. Binzel, T. Gehrels, \& M. S. Matthews (Tucson: Univ. Arizona Press), 240

Hagen, J. G. 1892, AJ, 12, 145

Hahn, J. M., \& Rettig, T. W. 2000, Icarus, 146, 501

Hawkins, G. S., Southworth, R. B., \& Steinon, F. 1959, AJ, 64, 183

Hergenrother, C. W., Spahr, T. B., Nelson, M., Gleason, A. E., \& Nakano, S. 2001, IAU Circ. 7635

Herrick, E. C. 1863, Sur les Etoiles Filantes (Brussels: Impr. M. Hayez)

Holetschek, J. 1909, Astron. Nachr., 179, 221

Hubbard, J. S. 1854, AJ, 4, 1

Hughes, D. W. 2003, MNRAS, 346, 584

Jenniskens, P. 1995, A\&A, 295, 206

2004, AJ, 127, 3018

. 2006, Meteor Showers and Their Parent Comets (Cambridge: Cambridge Univ. Press)

Jenniskens, P., \& Lyytinen, E. 2005, AJ, 130, 1286
Katasev, L. A., \& Kulikova, N. V. 1981a, Sol. Syst. Res., 14, 133 1981b, Sol. Syst. Res., 14, 179

Kronk, G. W. 1988, Meteor Showers: A Descriptive Catalog (Hillside: Enslow) . 1999, Cometography: A Catalog of Comets (Cambridge: Cambridge Univ. Press)

Lynn, W. T. 1905, Observatory, 28, 423

Makhmudov, N. 1982, Akad. Nauk SSSR Dokl., 25, 527

Marsden, B. G. 1972, Catalogue of Cometary Orbits (1st ed.; Cambridge: Cambridge Univ. Press)

Marsden, B. G., \& Sekanina, Z. 1971, AJ, 76, 1135

Newton, H. A. 1894, PASP, 6, 30

Nogami, N. 1995, Earth Moon Planets, 68, 435

Olivier, C. P. 1925, Meteors (Baltimore: Williams \& Wilkins)

Prentice, J. P. M. 1941, J. Brit. Astron. Assoc., 51, 92

Reznikov, E. A. 1982, in Meteornoe Veshchestvo v Mezhplanetnom Prostranstve, ed. O. I. Bel'kovich \& P. B. Babadzhanov (Moscow: Kazan), 151

Schulhof, L. 1894, Bull. Astron., 11, 126

Spratt, C. E. 1984, J. R. Astron. Soc. Canada, 78, 251

Sykes, M. V., \& Walker, R. G. 1992, in Asteroids, Comets, Meteors 1991, ed. A. W. Harris \& E. Bowell (Houston: LPI), 587

Trigo-Rodríguez, J. M., Betlem, H., \& Lyytinen, E. 2005, ApJ, 621, 1146

Vaubaillon, J. 2004, Ph.D. thesis, Inst. Méch. Céleste Calcul Éphémérides, Obs. Paris

Vaubaillon, J., Colas, F., \& Jorda, L. 2005, A\&A, 439, 761

Whipple, F. L. 1951, ApJ, 113, 464

Wiegert, P. A., Brown, P. G., Vaubaillon, J., \& Schijns, H. 2005, MNRAS, 361, 638

Williams, I. P. 2004, WGN Meteors, 32, 11

Wood, J. 1980, Radiant, 2, 95 